\title{
TECNOLOGIA “TELHADO VIVO”: ESTUDO E POTENCIAL DE INTEGRAÇÃO DE PLANTAS NATIVAS NA COBERTURA E RESGATE DA FAUNA
}

\author{
Isis Arend da Silva (PUC-RS, Brasil) isisarend@gmail.com \\ Patrícia Arbo Souza (PUC-RS, Brasil) patrícia.souza@pucrs.br \\ Sandro Rafael Dornelles Avila (PUC-RS, Brasil) sandro.avila@acad.pucrs.br \\ Tayra Wagner Zucchett (PUC-RS, Brasil) tayrawz@yahoo.com.br \\ Márcio Rosa D`Avila (PUC-RS, Brasil) marcio.davila@pucrs.br
}

Resumo: As áreas urbanas atualmente apresentam uma série de impactos ambientais, provenientes da densidade dos espaços construídos e a falta de qualidade ambiental nas cidades. $O$ objetivo da pesquisa é adaptar a tecnologia "Telhado Vivo" à região de execução, Porto Alegre e área metropolitana no Estado do Rio Grande do Sul/Brasil, por meio da utilização de espécies de vegetação nativa, viabilizando melhora na qualidade de vida dos moradores de áreas urbanas e o resgate da fauna e flora nativas. A pesquisa em curso é desenvolvida em dois projetos pilotos executados em escala real no Prédio $1 \mathrm{e}$ no Prédio 5, e, em escala experimental, no Canteiro Experimental da Faculdade de Arquitetura e Urbanismo, localizados no campus central da Pontifícia Universidade Católica do Rio Grande do Sul (PUCRS). O estudo é desenvolvido por meio de listas de vegetação nativa da região, livros, artigos e coleta em campo. Apresenta resultados parciais satisfatórios obtidos por meio da observação, da análise termográfica, do desenvolvimento, adaptação e integração da flora e fauna na tecnologia "Telhado Vivo".

Palavras-chave: Telhado verde. Arquitetura sustentável. Espécies nativas. Substrato. Elementos construtivos. Paisagismo.

Abstract: Nowadays, there are several environmental impacts in urban areas, due to the density of built spaces and the lack of environmental quality in cities. The objective of this research is to adapt the technology "Green Roof" to the execution region, Porto Alegre City and its metropolitan area, in the state of Rio Grande do Sul, Brazil, through the use of native vegetation species, making viable the improvement in the quality of life for the urban residents as well as the recovery of native flora and fauna. The ongoing research is developed in two pilot projects performed in real scale, at the Building 1 and Building 5 and, in experimental scale, in the Experimental Garden of the Architecture and Urban Planning College, which are located in the central campus of the Pontifícia Universidade Católica do Rio Grande do Sul (PUCRS). This study is developed by native vegetation reports of the region, books, articles and fieldwork collections. This paper presents partial satisfactory results which were got from the observation, thermographic analysis, development, adaption and integration of the flora and fauna in the technology of "Green Roof".

Keywords: Landscaping. Sustainable architecture. Green roof. Native species. Substratum. Constructive element. 


\section{Introdução}

A tecnologia "Telhado Vivo" pode ser descrita como uma cobertura para edificações (prédios, garagens, guaritas, paradas de ônibus e outras construções). Sua função apresenta grande potencial no contexto estético, paisagístico e eficiência energética da edificação. Tem capacidade de armazenamento de carbono (GETTER, 2009), otimização da temperatura na edificação nos períodos de inverno e verão, auxiliando na redução do consumo energético para o condicionamento artificial da edificação (CASTLETON, 2010; FANG, 2008; ROSA D' AVILA, 2010). No contexto do planejamento urbano essa tecnologia apresenta uma contribuição na redução e retardo das águas pluviais nos sistemas de escoamento e drenagem da cidade, não sendo suficiente em locais com altas taxas pluviais (BERNDTSSON, 2010; GREGORIE; CLAUSEN, 2011) e redução do fenômeno "Ilha de Calor" (TAKEBAYASHI; MORIYAMA, 2007). Outra característica é a melhora da qualidade do ar, já conhecido benefício de árvores, porém isto pode ser alcançado também por espécies de menor porte como as utilizadas na tecnologia "Telhado Vivo Extensivo" (YANG; YU; GONG, 2008; CURRIE; BASS, 2008) e para a redução de ruídos externos, cada vez mais presentes na vida dos moradores de áreas urbanas (RENTERGHEM; BOTTELDOOREN, 2009).

O modelo construtivo para a tecnologia "Telhado Vivo" é determinado por meio de seus objetivos: estético, com ou sem acesso de pessoas; disponibilidade de manutenção e construção nova ou já existente. São dois os sistemas construtivos da tecnologia "Telhado Vivo": extensivo e intensivo. Telhados extensivos constituem-se de uma camada fina de substrato, equivalente entre 5 e $15 \mathrm{~cm}$, tornando-o assim um telhado mais leve. Esse permite apenas cobertura vegetal de pequeno porte e resistentes ao clima da região, sendo comum o uso de gramíneas ou Sedum, o que não permite o acesso devido as suas raízes superficiais. Geralmente estes telhados são mais sustentáveis, uma vez que em alguns casos podem não requerer irrigação artificial e a sua manutenção é mínima, e também podem ser integrados a construções já existentes. Nos telhados intensivos usa-se uma camada mais profunda entre 15 e 120 centímetros de espessura de substrato, podendo suportar até algumas espécies de árvores. Sua estrutura permite o fluxo de pessoas, porém requer uma maior manutenção e sua estrutura requer planejamento prévio (ALMEIDA, 2008; KOSAREO, 2006). Alguns autores citam um terceiro modelo intermediário: tecnologia "Telhado Vivo" semi-intensivo, que teria uma altura de substrato intermediária e a vegetação utilizada poderia ser arbustiva.

Deve-se ter cuidado na escolha da vegetação como elemento construtivo na produção da tecnologia "Telhado Vivo". Estudos de seleção de espécies vegetais permitem que a cobertura verde instalada na tecnologia obtenha sucesso em condições adversas. E, das plantas mais utilizadas, destaca-se o gênero Sedum, por ter suas características muito estudadas e 
difundidas na Europa, continente de origem da maior parte das espécies do gênero citado. Entre suas características se destacam a resistência a dessecação e sobrevivência em solo raso. Porém, o uso de vegetação exótica em áreas urbanas pode causar perda de biodiversidade local, como: a extinção, mudanças genéticas e morfológicas e, alteração no padrão de reprodução de espécies. Sendo esta a segunda maior ameaça mundial a biodiversidade (ZILLER, 2001), desta forma contribuindo a intensificação de problemas ambientais já existentes. Para que a tecnologia "Telhado Vivo" realmente possa se adequar a região e contribuir na preservação do ecossistema local, é de suma importância a utilização de espécies nativas.

Neste estudo salienta-se a necessidade de alternativas sustentáveis em prol de melhoria da qualidade do ambiente urbano e promover a conservação da fauna e flora, por meio da caracterização de centros urbanos e, como os humanos influenciam neste ambiente. Descreve como a pesquisa foi desenvolvida, o uso de materiais e métodos utilizados. Analisa os resultados de como se deu o desenvolvimento, adaptação e desempenho quanto a contribuição da tecnologia "Telhado Vivo" no resgate da fauna e flora nos centros urbanos, objetivo deste estudo. Observa, também, as questões relacionadas ao equilíbrio térmico que visa enfatizar o sucesso da tecnologia e, também a importância da escolha da vegetação a ser utilizada. Os principais resultados e possíveis soluções para o problema de pesquisa são apresentados ao final deste estudo.

\section{Revisão literária}

Os seres humanos constroem seu habitat intervindo e transformando o meio natural. Esse processo de ocupação vem desencadeando um distanciamento do ser humano da natureza, perda de sua relação e conhecimento sobre a mesma, assim como a degradação da qualidade de vida no meio urbano (MORAN, 2008; GUERRA; CUNHA, 2001). Conforme Santos (2007: p. 5):

"Todos os empreendimentos voltados para a vida do homem, via de regra, se sobrepõem aos espaços e arranjos antes formados pelos elementos naturais, interferindo, de maneira contundente, nas relações e dinâmicas antes estabelecidas. Este fato é facilmente constatado pelo rastro de degradação e pelo comprometimento das funções ecológicas que hoje observamos".

Hoje os centros urbanos sofrem com uma série de impactos ambientais decorrentes da negligência com a qualidade ambiental urbana, como por exemplo: enchentes; movimentos de massa; poluição do ar e sonora; má qualidade da água e modificações climáticas como o fenômeno "ilha de calor". Segundo Huang (2008), Gomes, Gaja e Reig (1998) esse fenômeno favorece o aquecimento global, o que faz com que as demandas por tecnologias inovadoras em prol da sustentabilidade em todas as áreas aumentem, inclusive nas áreas como da arquitetura, urbanismo e paisagismo.

A flora nos centros urbanos não é apenas para efeito paisagístico, mas de regulação, pois 
contribui para uma melhor umidade do ar e temperatura no meio urbano por meio de processos de evapotranspiração, assim como, representa um filtro contra a poluição, contribuindo para a qualidade do ar. Outro papel da flora é servir como moradia e alimentação para a fauna, mantendo assim o equilíbrio biológico dos espaços construídos (GOMES, 1998, HAAREN; REICH, 2006; PENA, 2010) e pode ser inserido no meio urbano por meio da concepção de pequenas unidades de habitat como: praças, jardins, áreas de proteção, corredores e "stepping stones", ou trampolins ecológicos como, por exemplo, a tecnologia "Telhado Vivo".

As experiências apontam que ao longo dos anos foi se perdendo o conceito de construções que integrassem vegetação como elemento construtivo no projeto arquitetônico, privilegiando sistemas construtivos convencionais. No ano de 1990, quando questões ecológicas se tornaram evidentes no cenário mundial, a tecnologia "Telhado Vivo" passou a ser novamente resgatada como uma proposta mais sustentável na área da construção civil (ROSA D' AVILA, et al., 2010). O objetivo é contribuir para a redução do impacto do processo de urbanização desenfreado e o consequente aquecimento global devido, principalmente, ao processo de desmatamento e emissão de CO2 (OJIMA; HOGAN, 2008).

\section{Metodologia de pesquisa}

Os resultados parciais do estudo em andamento apresentam o desenvolvimento, adaptação, desempenho e a contribuição da tecnologia "Telhado Vivo" no resgate da fauna e flora nos centros urbanos. $\mathrm{O}$ projeto está sendo desenvolvido por meio do acompanhamento e análise da tecnologia executada na cobertura de duas edificações localizadas no Campus Central da PUCRS - Prédio 1 (Faculdade de Filosofia e Ciências Humanas). E prédio 5 (Reitoria), assim como, por meio de investigação de campo, pesquisa teórica e prática que orientam o estudo em curso.

\subsection{Biodiversidade e integração de} espécies vegetais nativas à tecnologia

"Telhado Vivos"

A pesquisa iniciou por meio de consultas de listas de vegetação nativa da região, considerando as características únicas para a sobrevivência da vegetação em coberturas. Então, as vegetações foram definidas a partir dos seguintes critérios: altura, profundidade das raízes (profundas e rasas), substrato (composição e espessura de substratos apropriados), resistência e desempenho em relação ao regime fluvial e insolação da região em estudo ${ }^{1}$. Com o objetivo de contribuir para o resgate da fauna no centro urbano definiu-se,

\footnotetext{
1 No processo de definição das espécies de vegetação, foram realizadas coletas em campo e testes realizados no Canteiro Experimental da Faculdade de Arquitetura.
} 
também, espécies frutíferas para sua atração. As aves, por exemplo, que fazem parte desta fauna, vem sofrendo com a redução de seu habitat, levando muitas espécies a extinção (FONTANA, 2005; PREFEITURA MUNICIPAL DE PORTO ALEGRE, 1975; BAUMAN, 2006).

Um estudo de biodiversidade foi realizado com o grupo dos Artrópodes, pois é o grupo mais numeroso de animais encontrados em todos os lugares do mundo. São provavelmente os primeiros animais terrestres a ter uma relação tão íntima com as plantas, um não vive sem o outro (GRIMALDI, 2001; TRIPLEHORN; JONNSON, 2011). O estudo foi realizado por meio de levantamentos fotográficos e armadilhas adesivas de cor amarela de $11.5 \mathrm{X}$ $8.5 \mathrm{~cm}$ (Figura 1), cedidas pelo Laboratório de Entomologia da PUCRS. Na execução da tecnologia "Telhado Vivo" no Prédio 1, apresentando uma área de cobertura de 475,9 $\mathrm{m}^{2}$, foram colocadas 3 armadilhas por coleta. Na execução da tecnologia no Prédio 5, com a área de cobertura de $118 \mathrm{~m}^{2}$, foi utilizado 2 armadilhas por coleta. As coletas foram executadas no mesmo período, na primeira coleta as armadilhas foram instaladas em 16/4/2012 e recolhidas em 24/4/2012. Na segunda coleta as armadilhas foram instaladas em 05/6/2012 e recolhidas em 19/6/2012.

Após coleta dos artrópodes, esses foram armazenados em álcool com concentração de
$70 \%$ para posterior identificação ${ }^{2}$ A classificação foi realizada de acordo com Rafael et. al. (2012), Triplehorn (2011) e Young (1977). Os artrópodes foram identificados até o menor nível taxonômico possível: família, gênero e ocasionalmente espécie. Quando não identificados até espécie foram diferenciados por morfo espécie (Figura 2). Não foram identificados artrópodes com menos de $3 \mathrm{~mm}$, devido à dificuldade associada ao campo da entomologia (RAFAEL; AGUIAR; AMORIM, 2009; SCOTT; SUSAN; JENNIFER, 2012).

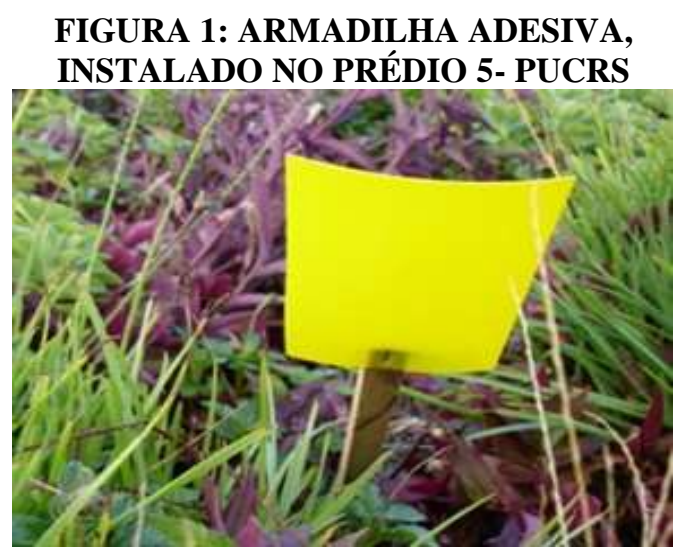

Fonte: Os autores

FIGURA 2: ATRÓPODES EM PROCESSO DE IDENTIFICAÇÃO

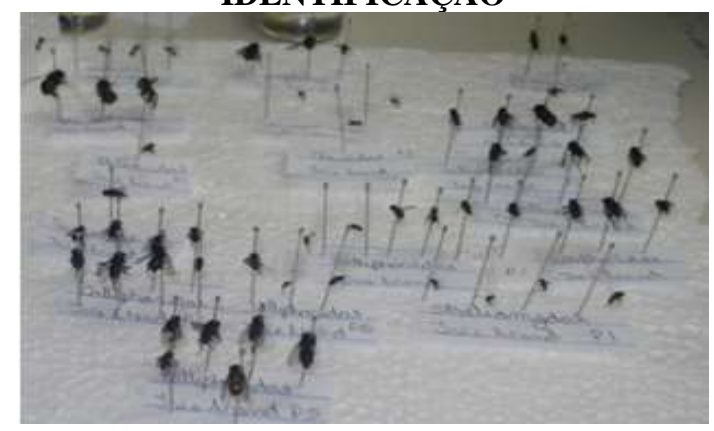

Fonte: Os autores

\footnotetext{
${ }^{2}$ Muitos artrópodes perdem a coloração com esse tipo de armazenamento, dificultando a identificação.
} 


\subsection{Investigação Termográfica Prédio 1 \\ (Reitoria)}

Com o intuito de reforçar a importância da vegetação na tecnologia "Telhado Vivo", o impacto desta no contexto urbano e melhor entendimento dos experimentos realizados no Canteiro Experimental, ao longo do experimento foram realizados estudos relacionadas à temperatura, umidade e precipitação (ROSA D' AVILA et al., 2010). No Prédio 1 (Reitoria, localizada no campus central PUCRS) foi realizado um estudo por meio de imagens termográficas com o aparelho "testo", na pós-execução da tecnologia, as imagens foram retiradas no dia 03 de janeiro de 2013.

\section{Discussão dos Resultados}

Após execução em escala real da tecnologia "Telhado Vivo" no Prédio 5 (Faculdade de Filosofia e Ciências Humanas, campus central PUCRS) foram realizadas entrevistas com usuários de escritórios adjacentes à área de intervenção. Os resultados apontam para o melhoramento do conforto térmico do ambiente adjacente, descartando-se o uso de equipamentos de condicionamento térmico artificial após execução da tecnologia. Constatado também, o efeito apaziguador da paisagem (ROSA D' AVILA et al., 2010). Um estudo foi realizado sobre a temperatura da edificação antes e pós-execução da tecnologia e, apontaram para um melhor desempenho térmico do ambiente (GARCIA FILHO et al., 2011).
A tecnologia "Telhado Vivo" executada na cobertura do Prédio 5 tem sido acompanhada desde 2010, por meio de levantamento fotográfico (Figura 3 e 4). Todas as vegetações obtiveram bom desempenho, não sendo necessários gastos com irrigação. A manutenção da tecnologia tem sido apenas uma poda anual das vegetações.

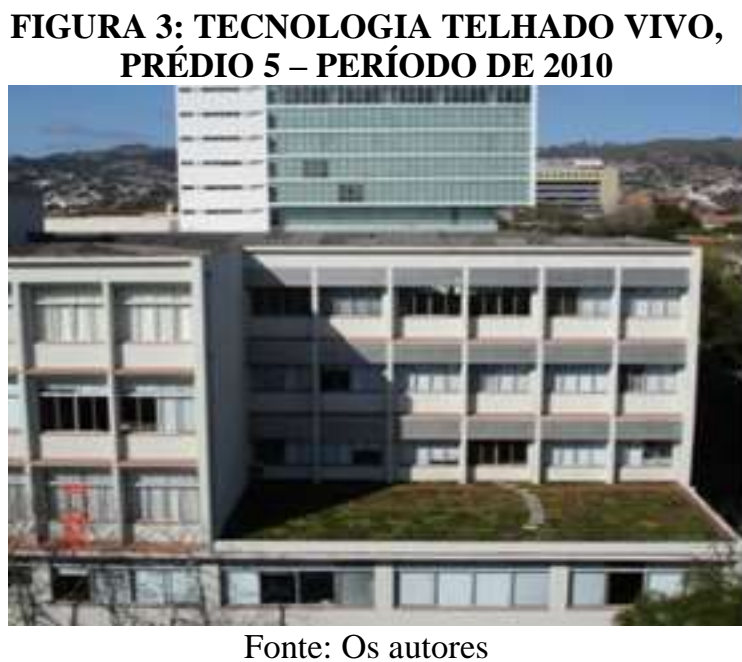

FIGURA 4: TECNOLOGIA TELHADO VIVO, PRÉDIO 5, PERÍODO DE 2012

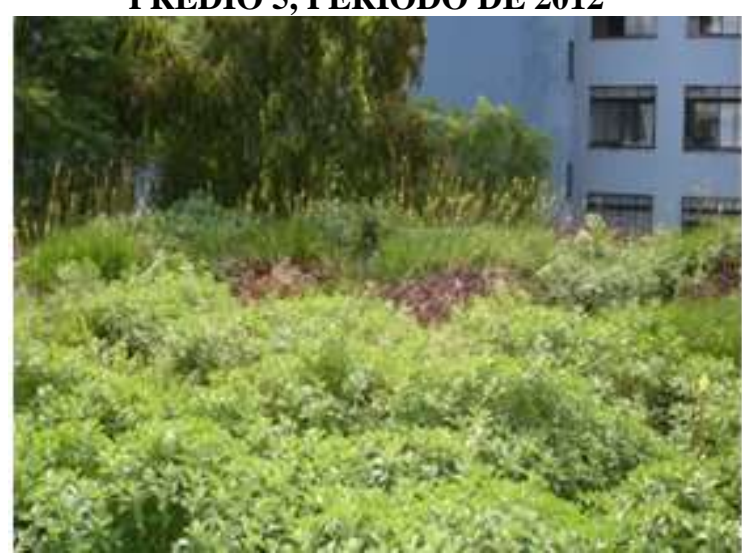

Fonte: Os autores

A tecnologia "Telhado Vivo" executada no Prédio 1 (Figuras 5 e $\quad 6$ ) está em acompanhamento desde 2012, logo após a sua execução. Todas as vegetações apresentaram um bom desenvolvimento, evitando também o uso de irrigação artificial. O uso de elementos 


\section{ReLAInEF?}

modulares dificultou a forração total da vegetação, podendo ser observado "falhas" ao longo da cobertura, módulos sem vegetação. $\mathrm{O}$ estudo termográfico identificou $14^{\circ} \mathrm{C}$ de diferença para áreas com vegetação e sem vegetação (apenas substrato na tecnologia) (Figuras 7, 8, 9 e 10). Como observado, a temperatura em um calçamento normal pode, neste dia, analisado em específico, a $41,1^{\circ} \mathrm{C}$ (Figura 12), enquanto que, com a tecnologia "Telhado Vivo" temos temperaturas de $21,2^{\circ}$ C (Figura 10), perfazendo uma diferença de $19,9^{\circ} \mathrm{C}$.

FIGURA 5: PRÉDIO 1 (REITORIA), SISTEMA

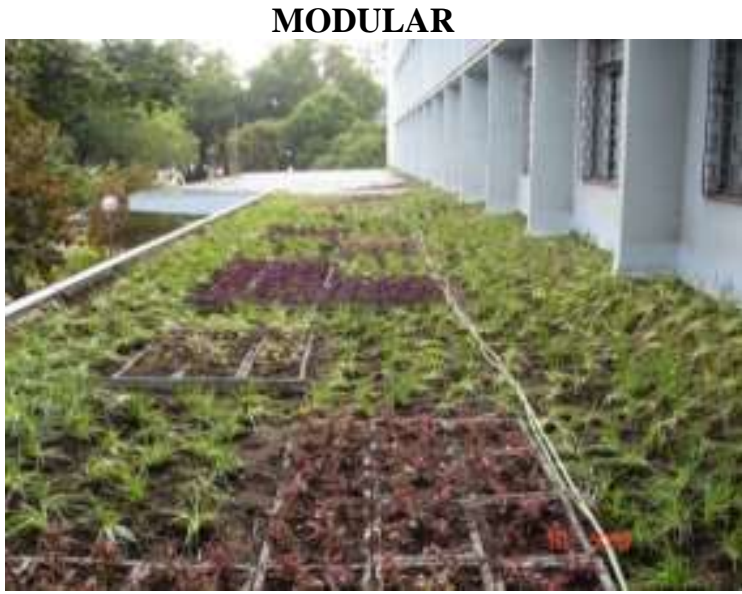

Fonte: Os autores

FIGURA 6: PRÉDIO 1 (REITORIA), SISTEMA MODULAR.

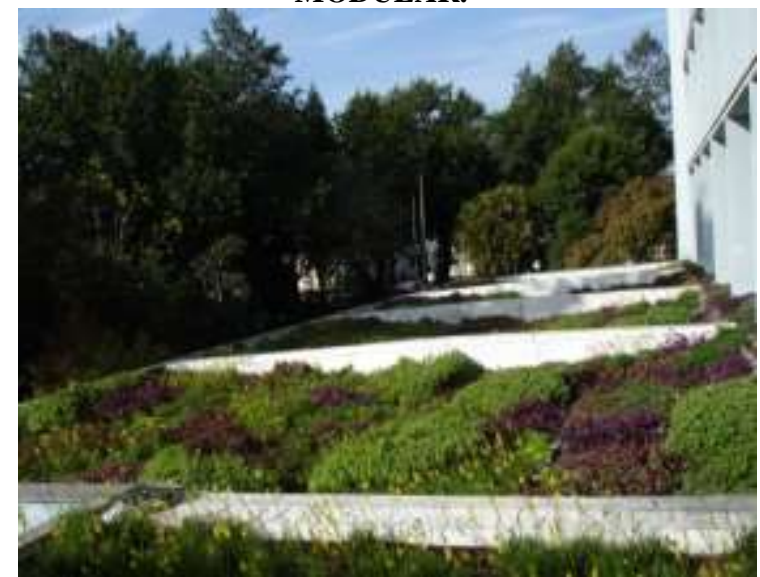

FIGURA 7: IMAGEM DA TECNOLOGIA TELHADO VIVO EXECUTADA NO PRÉDIO 1 (REITORIA)

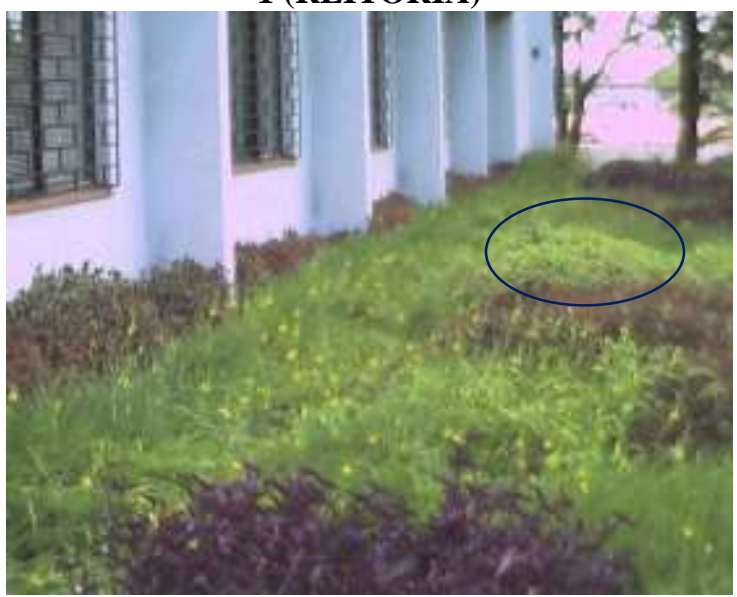

Fonte: Os autores

Figura 8: Imagem da tecnologia Telhado Vivo executada no Prédio 1 (Reitoria), campus central PUCRS, versão termográfica

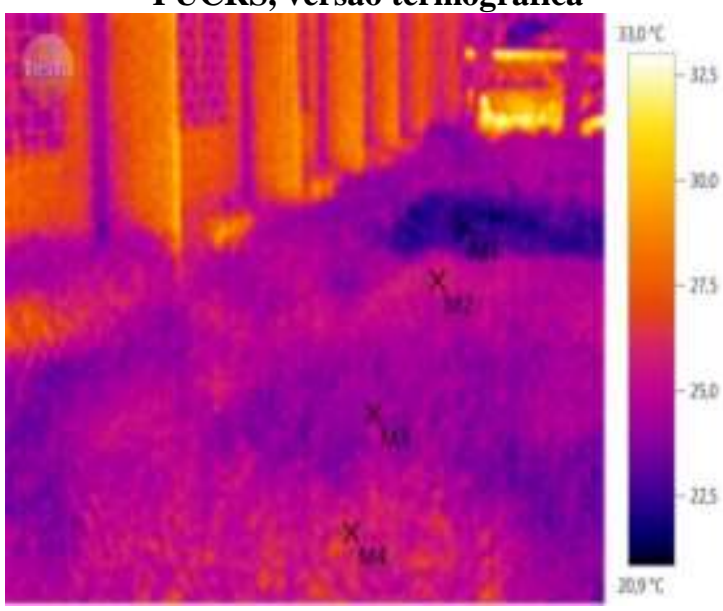

Fonte: Os autores

Em XM1, a Sphagneticola trilobata (L.) Pruski, com temperatura de $21,4{ }^{\circ} \mathrm{C}$, XM2 Tradescantia zebrina Heynh. ex Bosse, com temperatura de $26^{\circ} \mathrm{C}$, XM3 Bulbine frutescens (L.) Willd., com temperatura de $24,4{ }^{\circ} \mathrm{C}, \mathrm{XM} 4$ Tradescantia pallida purpurea (Rose) D. R. Hunt var, com temperatura de $26,4^{\circ} \mathrm{C}$. 


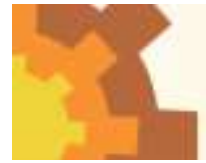

FIGURA 9: IMAGEM DA TECNOLOGIA TELHADO VIVO EXECUTADA NO PRÉDIO 1 (REITORIA), CAMPUS CENTRAL PUCRS

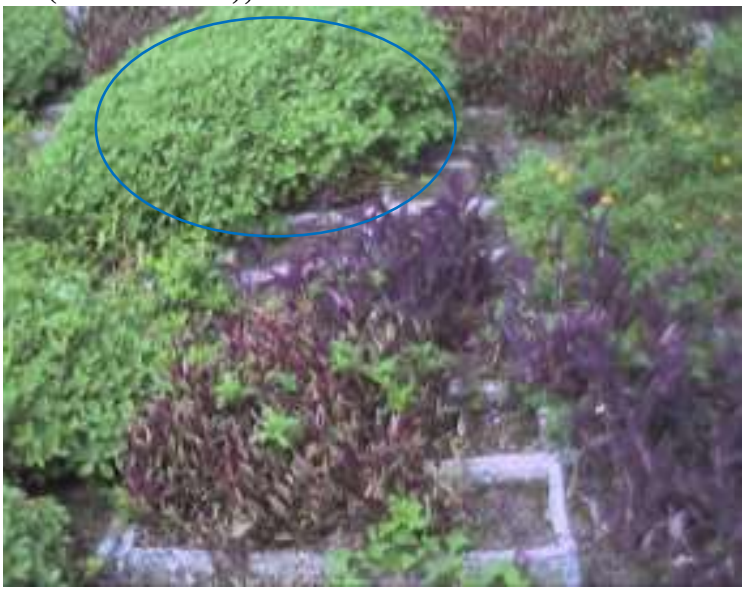

Fonte: Os autores

FIGURA 10: IMAGEM DA TECNOLOGIA TELHADO VIVO EXECUTADA NO PRÉDIO 1 (REITORIA), CAMPUS CENTRAL PUCRS, VERSÃO TERMOGRÁFICA

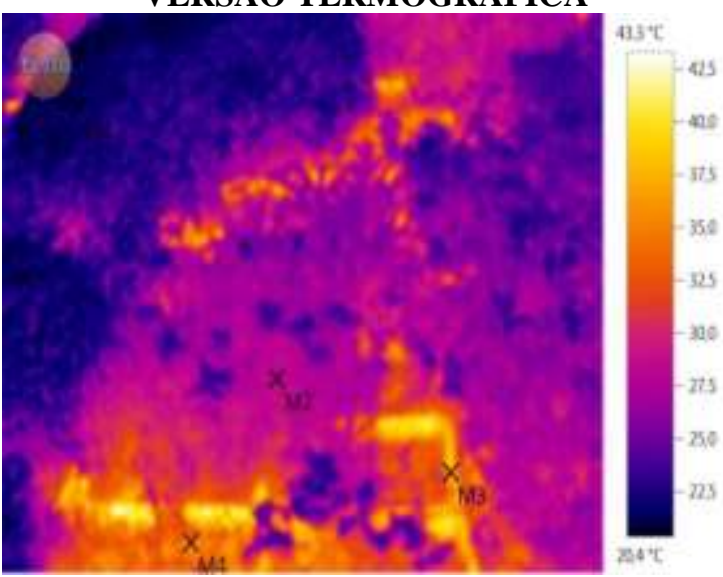

Fonte: Os autores

Em XM1 (circulado em laranja), a Plectranthus neochilus Schlechter, com temperatura de $21,2{ }^{\circ} \mathrm{C}$, XM2 Tradescantia zebrina Heynh. ex Bosse, com temperatura de $28{ }^{\circ} \mathrm{C}$, XM3 borda do elemento construtivo retangular utilizado com temperatura de 37,5 ${ }^{\circ} \mathrm{C}$, XM4 solo nu com temperatura de $35,4^{\circ} \mathrm{C}$.

\section{FIGURA 11: IMAGEM EM CORES DO EQUIPAMENTO TERMOGRÁFICO}

"TESTO", JARDIM E CALCAMENTO EM

FRENTE AO PRÉDIO 9 (FAU) CAMPUS

CENTRAL PUCRS

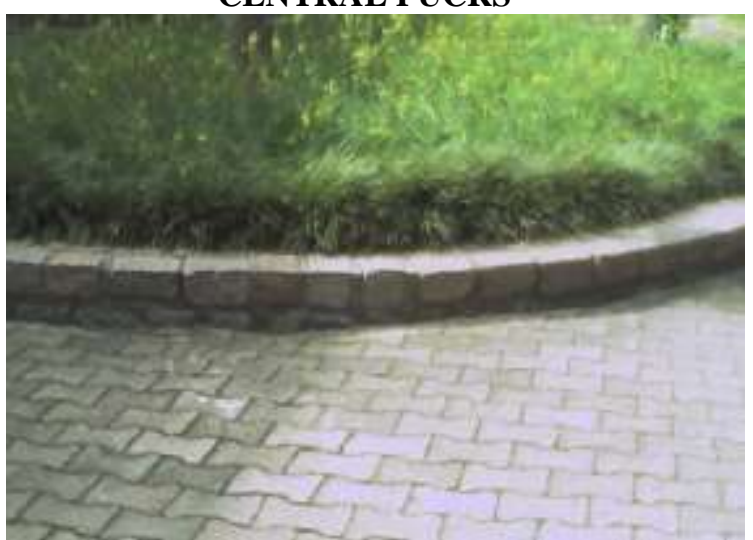

Fonte: Os autores

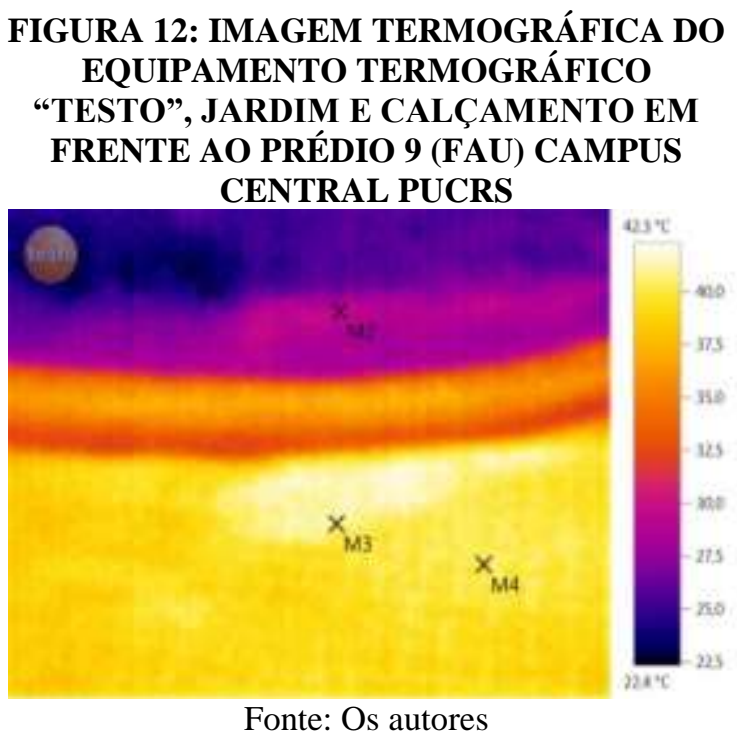

Em XM1 (circulado em laranja) a espécie Bulbine frutescens (L.) Willd. com a temperatura de $22,6{ }^{\circ} \mathrm{C}, \mathrm{XM} 2$ a espécie Ophiopogon japonicus (L.f.) Ker Gawl. com temperatura de $29.4{ }^{\circ} \mathrm{C}$, XM3 calçamento com temperatura de $41,1{ }^{\circ} \mathrm{C}$ e XM4 calçamento com temperatura de $40,2{ }^{\circ} \mathrm{C}$.

É possível observar que algumas vegetações são mais eficientes na redução da temperatura devido a sua reflexão e capacidade de sombreamento. Na Figura 9, a Sphagneticola trilobata (L.) Pruski (uma espécie nativa 


\section{ReLAInEF}

integrada à tecnologia) conhecida como Vedélia, tem a menor temperatura superficial do que as outras espécies. Essa espécie está sendo utilizada em ambos os Prédios 1 (Reitoria) e 5 (FFCH). Na Figura 11 a espécie Plectranthus neochilus Schlechter tem a menor temperatura em relação a outras espécies.

Para a melhor adequação da tecnologia e por motivos de preservação das espécies foi elabora uma lista de 50 espécies nativas que tem o potencial de ser integrada à tecnologia. Estas espécies estão distribuídas em 22 famílias botânicas.

Algumas espécies possuem características medicinais como a Baccharis articulata (Lam.) Pers (carqueja) (Figura 13), usada popularmente como digestiva, diurética, amarga, tônica, antifebril e anemia (SIMÕES et. al., 1998); frutífera como a Psidium incanum (O.Berg) Burret (araçá-do-campo) (Figura 14); atração a fauna como a Ruellia brevifolia (Pohl) C.Ezcurra (pingo-de-sangue) (Figura 15) que de acordo com Sigrist e Sazima (2002) esta espécie tem 20 possíveis espécies polinizadoras distribuídas entre a classe das aves, e as ordens Hymenoptera e Lepidoptera ambas, Artrópodes. Seus principais polinizadores são: Amazilia lactea (beija-flor), Heliconius etilla narcaea e Phoebis argante (borboletas).
FIGURA 13: BACCHARIS ARTICULATA

(LAM.)

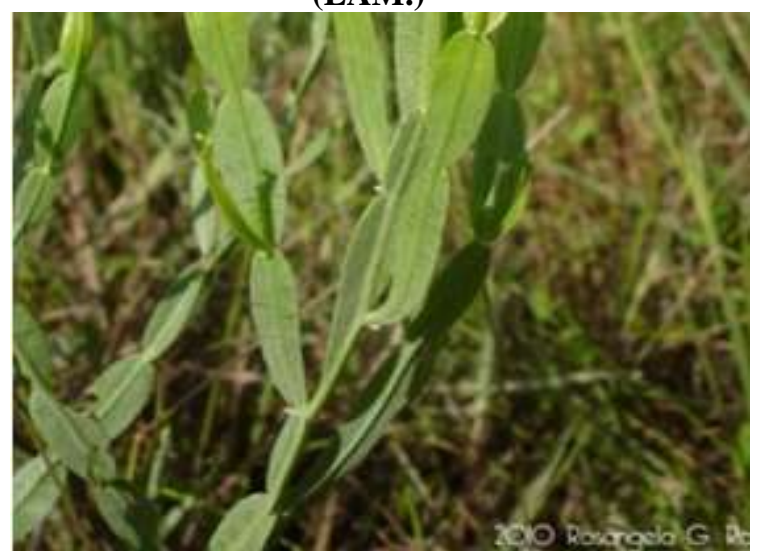

Fonte: Rosângela Gonçalves

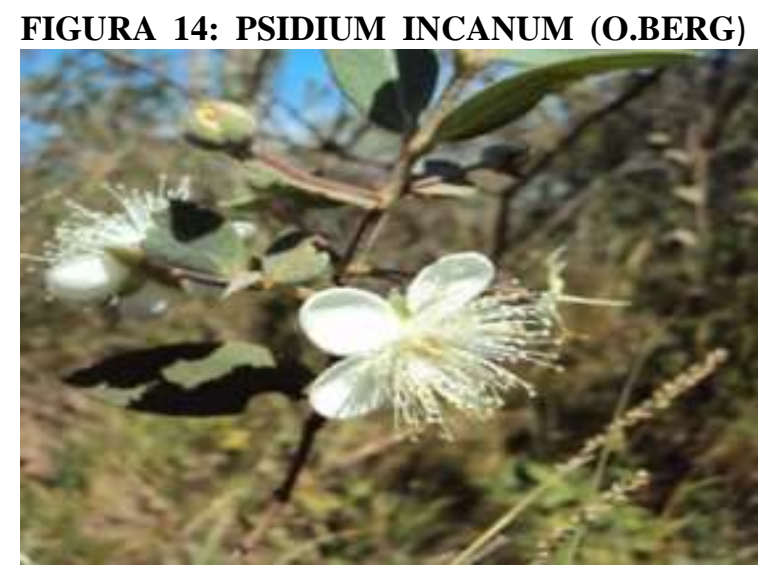

Fonte: Luciana Mancino

FIGURA 15: RUELLIA BREVIFOLIA (POHL) C.EZCURRA

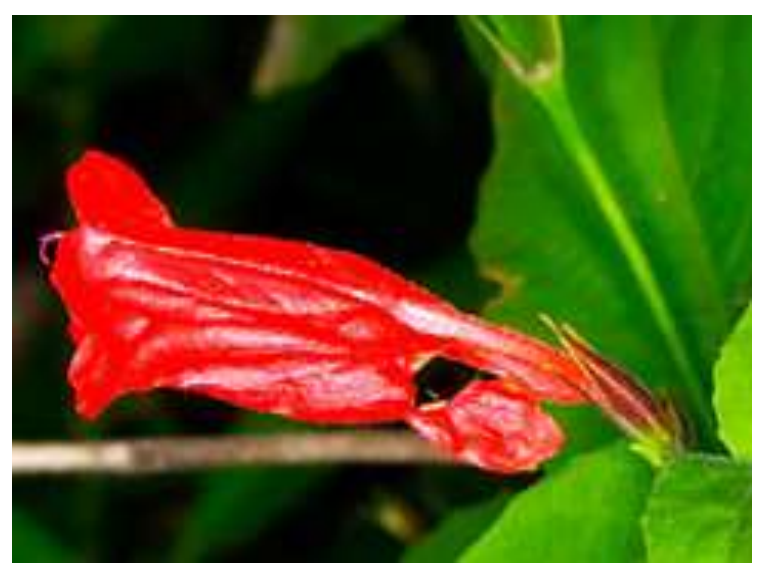

Fonte: Keoki Stender

Em ambos os prédios 1 (Reitoria) e 5 (Faculdade de Filosofia e Ciências Humanas) há em sua constituição florística a espécie Sphagneticola trilobata (L.) Pruski (Figuras 17 


\section{ReLAInEF}

e 18). Essa espécie tem se adaptado satisfatoriamente e também possui propriedades medicinais. Seu princípio ativo deriva do ácido caurenóico (KA), que possui como propriedades biológicas: analgésico, tripanossomicida, antifúngica, relaxante muscular, anti-inflamatório e propriedades hipoglicemiantes (FUCINATA et al., 2012; VENDRUSCOLO; MENTZ, 2006).

Em 2012 começou a ser realizada a pesquisa com os Artrópodes. Ao longo das coletas foram identificadas 58 morfoespécies, distribuídas em 7 ordens: Diptera (moscas e mosquitos), Hymenoptera (abelhas, vespas e formigas) (figura 16), Hemiptera (cigarrinhas), Lepidoptera (borboletas e mariposas) (figura 17), Coleoptera (besouros), Odonata (libélulas), Areneae (aranhas). Diptera foi a ordem com maior número de indivíduos e espécies. Algumas espécies identificadas como a Oncometopia facialis (SIGNORET, 1854), conhecida como cigarrinha-do-citros, por exemplo, é considerada uma praga tanto para as plantações de citros quanto para a de café (GALLO, et. al., 2002).

Mas, também situação contrária foi detectada, por exemplo, a espécie Cycloneda sanguinea (LINNAEUS, 1743) (Figura 18), muito comum no Brasil e utilizada para controle de pragas nas plantações de algodão (MINKS; HARREWIJN, 1988; GALLO, 2002). Foi incrível a biodiversidade encontrada na tecnologia, mesmo com o curto período de coletas e a limitação das armadilhas utilizadas. Também foi encontrada uma possível nova espécie de cigarrinha na tecnologia. A espécie encontrada foi um Bucephalogonia sp., existe apenas uma espécie registrada para este gênero e, o indivíduo capturado não se enquadra as características morfo anatômicas, por ser uma fêmea. Então será necessário a captura de novos indivíduos para se obter a determinação correta. Salienta-se que apenas um indivíduo foi encontrado, espécies únicas (coletadas apenas uma única vez) são frequentemente capturadas acidentalmente, possivelmente não frequentam a tecnologia.

FIGURA 16: BOMBUS SP. ABELHA PERTENCENTE À ORDEM HYMENOPTERA

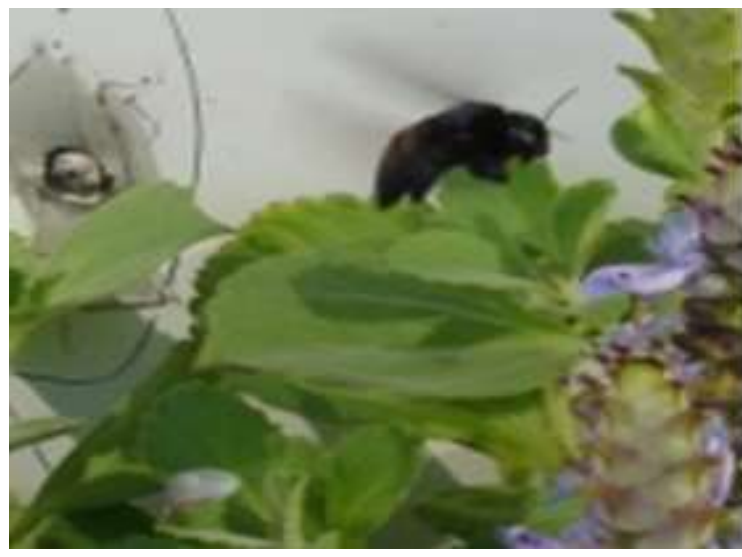

Fonte: Schlechter

FIGURA 17: INDIVÍDUO PERTENCENTE À ORDEM LEPIDOPTERA

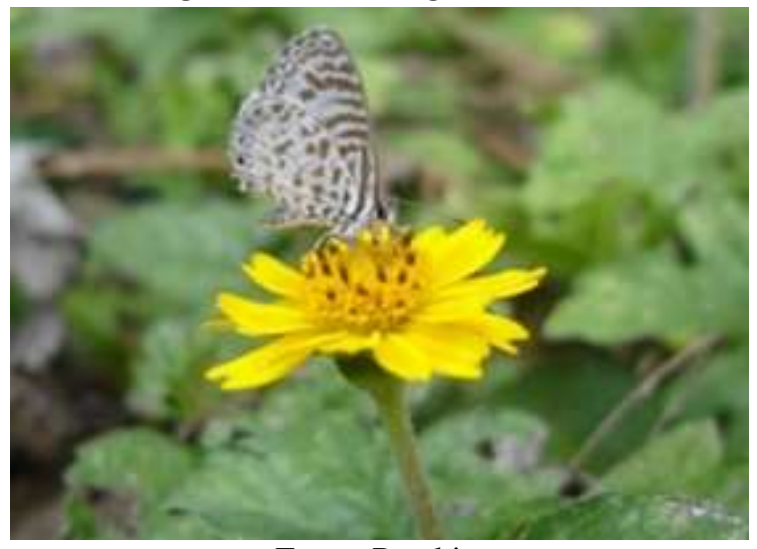

Fonte: Pruski 
FIGURA 18: CYCLONEDA SANGUINEA

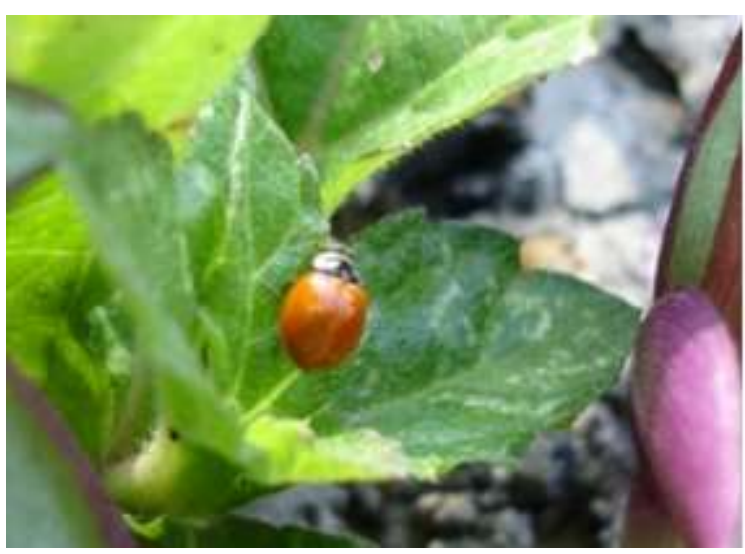

Fonte: Linnaeus, 1743

As aves também foram beneficiadas pela tecnologia. A ave Troglodytes musculus (NAUMANN, 1823), conhecido como corruíra, faz visitas constantes a tecnologia, essa espécie tem sua alimentação composta por pequenos frutos e invertebrados (BELTON, 1994; EFE, 2001; RASQUIM). Outras espécies não foram avistadas, principalmente, pela falta de espécies frutíferas na tecnologia.

Apesar do curto tempo de acompanhamento é possível concluir que se formou uma comunidade estável e equilibrada de artrópodes na tecnologia “Telhado Vivo". Com o crescente processo de perda de áreas verdes e perda de fauna nas cidades, se observa a necessidade de maior disponibilidade de alimento para fauna. E, por meio do acompanhamento e estudo da comunidade de artrópodes se confirmou o pleno uso desta nova área de flora como recurso alimentar e, também, de refúgio de algumas espécies. Serviços de ciclagem, polinização e fornecimento de alimento são observados na tecnologia o que demonstra a aceitação e integração desta área nas comunidades animais. Ao integrar vegetações nativas na tecnologia, garantem-se os serviços ecológicos prestados por este grupo, indispensáveis para a vida e aumento da distribuição destas vegetações nas áreas urbanas e rurais.

A fauna de maior porte como as aves, apesar de adaptadas ao ambiente urbano, muitas espécies estão deixando de ser avistadas em certas regiões. Há uma grande dificuldade para encontrar alimentos adequados e em quantidade, além de lugares para a nidificação. A presença de apenas uma espécie de ave que visita esporadicamente à tecnologia, reforça a necessidade de espécies de vegetação indígena que as atraiam, principalmente, as espécies frutíferas.

\section{Considerações finais}

Esta pesquisa aponta para o sucesso da tecnologia "Telhado Vivo" quanto ao melhoramento do conforto térmico da edificação e como uma ferramenta de preservação, tendo obtido resultados positivos quanto a integração de fauna e flora nativas. Quanto ao conforto térmico, foi constado o descarte no uso de condicionamento artificial ao ambiente adjacente a tecnologia, em estudo, no Prédio 5. Por meio de estudos termográficos também foi identificada uma diferença de $19,9^{\circ}$ C para um calçamento normal e a tecnologia "Telhado Vivo".

Ao longo do estudo de integração de espécies vegetais nativas desenvolveu-se uma lista com mais de 50 espécies vegetais nativas, com potencial para a substituição das espécies exóticas atualmente utilizadas na tecnologia. 
Como exemplo, pode-se citar a Sphagneticola trilobata (L.) Pruski, a qual já se encontrava presente na tecnologia executada em escala real na PUCRS, em ambos os Prédios 1 e 5. A espécie Sphagneticola trilobata (L.) Pruski apresenta as características necessárias para a utilização em telhados vivos e, como observado a espécie se provou integrada a tecnologia. Além de ter obtido o melhor resultado na contenção de raios solares, apresentando o valor mais baixo de temperatura entre as espécies presentes no Prédio 1 da PUCRS.

Ao se realizar o estudo de biodiversidade constatou-se que a comunidade animal se integrou com sucesso a tecnologia, onde estes encontram recursos alimentares extras e abrigo. Ao acompanhar o desenvolvimento da tecnologia foi possível observar a apropriação da fauna aos poucos e, também, a presença de serviços de ciclagem, polinização e fornecimento de alimento. Entre as espécies de artrópodes encontrados salienta-se uma possível nova espécie do gênero Bucephalogonia. Espécies de maior porte como aves ainda encontram dificuldade em encontrar alimento na tecnologia "Telhado Vivo" devido à falta de espécies frutíferas. A única espécie avistada foi a ave Troglodytes musculus (NAUMANN, 1823), conhecida como corruíra, que vai a busca de pequenos invertebrados.

Como sugestão para um estudo mais acurado de integração de fauna e flora nativa na tecnologia "Telhado Vivo", ainda será necessário o plantio e teste das espécies nativas com potencial de integração a esta tecnologia, a execução em escala real da tecnologia somente com a utilização destas espécies e, também a realização de estudos relacionados a produção de espécies nativas que possam ser utilizadas nas chamadas "stepping stones" ou corredores verdes.

\section{Agradecimentos}

Esta investigação faz parte do grupo de pesquisa Eficiência Energética na Arquitetura do CNPq, em curso desde 2009 com fomento da Fundação de Amparo à Pesquisa do Estado do Rio Grande do Sul (FAPERGS) por meio do programa de fomento à pesquisa Auxílio Recém Doutor, do Programa Institucional de Bolsas de Iniciação Científica do $\mathrm{CNPq}$ (PIBITICNPq) e da PUCRS. Agradeço a todas as instituições citadas por possibilitar esta pesquisa. Ao nosso orientador Prof. Dr. Márcio Rosa D'Avila, por nos receber como orientandos e nos ajudar a crescer como profissionais. Ao Prof. Dr. Gervasio Silva Carvalho, do Laboratório de Entomologia da PUCRS por ceder equipamentos e o laboratório para que fosse possível realizar a identificação dos artrópodes e, também aos seus bolsistas, Douglas Sebben, Jonas Podkowa e Jéssica Bohusch Oliseski, que participaram ativamente na determinação dos artrópodes. Ao Prof. Dr. Claudio Augusto Mondin (Faculdade de Ciências BiológicasPUCRS), pela ajuda no processo de escolha das espécies de vegetações nativas. 


\section{Referências}

ALMEIDA, M.A.M. Coberturas naturadas e qualidade ambiental: Uma contribuição em clima tropical úmido. Viii, 151 f. I1 Dissertação(mestrado) - Universidade Federal do Rio de Janeiro, PROARQ, 2008. Disponível em: http://teses2.ufrj.br/Teses/FAU_M/MarcoAnt onioMilazzoDeAlmeida.pdf. Acesso em: 08 set. 2011.

BAUMAN, N. Ground- Nesting Birds on Green Roofs in Switzerland: Preliminary Observations. Urban Habitats, v. 4, n.1. 2006. Disponível em: http: / / urbanhabitats.org/v04n01/birds_pdf.pdf $>$ Acesso em: 28 set. 2011.

BELTON, W. Aves do Rio Grande do Sul: Distribuição e biologia. São Leopoldo. Ed. UNISINOS, 1994. 584 P.

BERNDTSSON, J.C. Green roof performance towards management of runoff water quantity and quality: A review. Ecological Engineering, v. 36, n.4, p. 351-360. 2010. Disponível em: http://www.scopus.com/record/display.url?or igin $=$ recordpage $\&$ zone $=$ relatedDocuments\&eid $=2$-s2.0-

77049095557\&noHighlight $=$ false\&relpos $=1 \#$ Acesso em: 14 set. 2011.

CASTLETON, H.F. et al. Green roofs; building energy savings and the potential for retrofit. Energy and buildings, v. 42, n. 10, p. 15821591. 2010. Disponível em: <http://www.sciencedirect.com/science/articl e/pii/S0378778810001453> Acesso em: 14 set. 2011.

CURRIE, B.A.; BASS, B. Estimates of air pollution mitigation with Green plants and Green roofs using the UFORE model. Urban Ecosystems, v. 11, n. 4, p. 409-422. 2008. Disponível em: < http: / /www.scopus.com/record/display.url?ei $\mathrm{d}=2$-s2.0-56149100142\& origin $=$ reflist\#>

Acesso em: 16 set. 2011.

EFE, M.A.; MOHR, L.V.; BUGONI, L. Guia ilustrado das aves de Porto Alegre. Porto Alegre: PROAVES, SMAM, COPESUL, CEMAVE, 2001. 144p.

FANG, C. Evaluating the thermal redution effect of plant layers on rooftops. Energy and Buildings, v.40, n.8, p. 1048-1052. 2008.
Disponível

em:

$<$ http:/ / www.sciencedirect.com/science/article /pii/S0378778807002228> Acesso em: 15 set. 2011.

FOnTANA, C. S. A ornitofauna em Porto Alegre no século $\mathbf{X X}$ : status de ocorrência e conservação. Comunicação do Museu de Ciências e Tecnologia da PUCRS, série zoologia, v. 18, n. 2, p. 161-206. Dez. 2005.

FUCINATA G. et al. Development and validation of a stability indicative HPLCPDA method for kaurenoic acid in spray dried extracts of Sphagneticola trilobata (L.) Pruski, Asteraceae. Elsevier, v. 101, p. 530-536. 2012. Disponível em: < http: / / www.sciencedirect.com/science/article /pii/S0039914012007801 >. Acesso em: 04 dez. 2012.

GALLO, D., et. al. Entomologia Agrícola. Piracicaba: FEALQ, 2002. v. 10, 920p.

GARCIA FILHO, D. O. ; AVILA, S. R. D. ; ROSA, S. D. ; Cezar, R. O. ; ROSA D' AVILA, Márcio . Análise da Eficiência Energética, com Base nas Variações de Temperatura, Sobre a Tecnologia Telhado Vivo, Implantada no Prédio Cinco do Campus Central da PUCRS. In: XII Salão de Iniciação Científica da PUCRS, 2011, Porto Alegre. XII Salão de Iniciação Científica da PUCRS, 2011.

GETTER, K.L. et al. Carbon Sequestration Potential of Extensive Green Roofs. Environmental Science \& Technology, v. 43, n. 19, p. 7564-7570. 2009.

GOMES, F.; GAJA, E.; REIG, A. Vegetation and climatic changes in a city. Ecological Engineering, n. 10, p. 355-360, 1998.

GREGORIE, B.G;CLAUSEN, J.C. Effect of a modular extensive Green roof on stormwater runoff water quality. Ecological Engineering, v.37, n.6, p. 963-969. 2011. Disponível em: < http://www.sciencedirect.com/science/article /pii/S0925857411000693> Acesso em: 15 set. 2011.

GRIMALDI, D. Insect evolutionary hostory from handlirsch to hennid, and beyond. Journal of Paleontology, v. 75, n. 6, p. 1152-1160. Nov. 2001. Disponível em: < http: / / search.proquest.com/biologyjournals/do cview/231035051/fulltextPDF/13350E41AA0 
A2D01B0/1 ?accountid $=8034>$ Acesso em: 22 nov. 2011.

GUERRA, A.J.T.; CUNHA, S.B. (organizadores). Impactos ambientais urbanos no Brasil. Rio de Janeiro: Bertrand, Brasil. 2001. 416p.

HAAREN, C.V.; REICH M. The German way to greenways and habitat networks. Landscape and Urban Planning, v. 76, n. 1-4, p. 7-22. Hannover, Germany, 2006. Disponível em: <http://www.sciencedirect.com/science/a rticle/pii/S0169204604001318> Acesso em: 24 out. 2011

HUANG, Y. et al. A fieldwork study on the diurnal changes of urban microclimate in four types of ground cover and urban heat island of Nanjing, China. Building and Environment, v. 43, n. 1, p. 7-17. 2008. Disponível em: <http://www.sciencedirect.com/science/articl e/pii/S0360132306004446\#bibl001> Acesso em: 24 out. 2011.

KOSAREO, L. Comparative environmental life cycle assessment of green roofs. Building and Environment, v.42, n. 7, p. 2606-2613. 2006. Disponível em: <http://www.sciencedirect.com/science/articl e/pii/S0360132306001648> Acesso em: 12 set. 2011.

MINKS, A. K. e HARREWIJN P. Aphids: Their Biology. Natural Enemies and Control. In: World Crop Pests.v. 2B. Amsterdam: Elservier. 1988. 364 p.

MORAN, E.F. Nós e natureza: uma introdução ás relações homem-ambiente. Editora Senac, São Paulo, 2008. 302 p.

OJIMA R, HOGAN D.J. População, urbanização e ambiente no cenário das mudanças ambientais globais: debates e desafios para a demografia brasileira. 2008. Disponível em: <http://www.abep.nepo.unicamp.br/encontro 2008/docspdf/abep2008_1383.pdf> Acesso em: 13 set. 2011.

PENA, S.B. et al. A methodology for creating greenways through multidisciplinary sustainable landscape planning. Journal of Environmental Management, v. 91, n. 4, p. 970-983. Lisboa, Portugal, 2010. Disponível em:<http://www.sciencedirect.com/science/a rticle/pii/S0301479709004010\# sec $>$ Acesso em: 24 out. 2011.

PREFEITURA MUNICIPAL DE PORTO ALEGRE. Plano piloto parque Saint' Hilaire. 1975.

RAFAEL, J.A et al. Insetos do Brasil: Diversidade e Taxonomia. Ribeirão Preto. Ed. Holos, 2012. 810 p.

RAFAEL, J.A.; AGUIAR, A.P. e AMORIM, D.S. Knowledge of insect diversity in Brasil: challenges and advances. Neotropical Entomology, v. 38, n. 5, 565-570 p. 2009. Disponível em: <http: / / www.scielo.br/scielo.php?pid=S1519566X2009000500001\&script $=$ sci_arttext $>$

Acesso em: 10 ago. 2012.

RASQUIM, U.B e FONTANA, C.S. Guia Eletrônico das Aves do Campus Central. Disponível em: <http://www.pucrs.br/mct/avifauna/index.ht $\mathrm{ml}>$. Acesso em: 26 jul. 2012.

RENTERGHEM, T.V; BOTTELDOOREN, D. Reducing the acoustical façade load from road traffic with green roofs. Building and Environment, v.44, n. 5, p. 1081-1087. 2009. Disponível em:

http:/ / www.sciencedirect.com/science/article /pii/S0360132308001923> Acesso em: 16 set. 2011.

ROSA D' AVILA, Márcio; Fritscher, Juliana Pasquetti C.; Peralta, Êmili S. Cobertura Vegetal: desenvolvimento e produção da tecnologia Telhado Vivo. In: Congresso Internacional Sustentabilidade e Habitação de Interesse Social - CHIS 2010, 2010, Porto Alegre. Congresso Internacional Sustentabilidade e Habitação de Interesse Social - CHIS 2010. Porto Alegre: ediPUCRS, 2010.

ROSA D' AVILA, Márcio; Cunha, Sabrina Cestari Correa da; Fritscher, Juliana Pasquetti C.; Peralta, Êmili S. Sustentabilidade na Edificação: Estudo e Execução da Tecnologia Telhado Vivo na Região Metropolitana de Porto Alegre. In: 54th World Congress IFHP 2010 Porto Alegre, Building Communities for the Cities of the Future, 2010, Porto Alegre. 54th World Congress IFHP 2010 Porto Alegre, Building Communities for the Cities of the Future. Porto Alegre: ediPUCRS, 2010.

ROSA D' AVILA, Márcio; Peralta, Êmili S.; 
Fritscher, Juliana Pasquetti C.; Cunha, Sabrina Cestari Correa da. Exposição no Museu de Ciência e Tecnologia da PUCRS Tecnologia Telhado Vivo: apresentação do projeto de pesquisa dos estudos por meio de painéis e elementos prototípicos. In: 54th World Congress IFHP 2010, Porto Alegre, Building Communities for the Cities of the Future, 2010, Porto Alegre. 54th World Congress IFHP 2010 Porto Alegre, Building Communities for the Cities of the Future. Porto Alegre: JOAObn, 2010 .

ROSA D' AVILA, Márcio; Fritscher, Juliana Pasquetti C.; Peralta, Êmili S. Cobertura Vegetal: desenvolvimento e produção da tecnologia Telhado Vivo. In: Congresso Internacional Sustentabilidade e Habitação de Interesse Social - CHIS 2010, 2010, Porto Alegre. Congresso Internacional Sustentabilidade e Habitação de Interesse Social - CHIS 2010. Porto Alegre: ediPUCRS, 2010.

SANTOS, R.F.(Org.). Vulnerabilidade Ambiental: Desastres naturais ou fenômenos induzidos? Brasilia: MMA, 2007, 192 p.

SCOTT, H.M.A.; SUSAN C.C.P.; JENNIFER S.T. Relationships between arthropod richness, evenness, and diversity are altered by complementary among plant genotypes. Oecologia, v. 168, n. 4, p. 1013-1021 p. 2012. Disponível em: <http:/ /www.springerlink.com/content/ulngk 72127744249/fulltext.pdf $>$ Acesso em: 03 jun. 2012.

SIGRIST, M.R. e SAZIMA, M. Ruellia brevifolia (Pohl) Ezcurra (Acanthaceae): fenologia da floração, biologia da polinização e reprodução. Revista Brasileira de Botânica, v. 25, n. 1, p. 35-42. 2002. Disponível em: < http: //www.scielo.br/pdf/\%0D/rbb/v25n1/a 06v25n1.pdf > Acesso em: 03 dez. 2012.

SIMÕES, C.M.O. et. all. Plantas da Medicina Popular no Rio Grande do Sul. Ed. Universidade/ UFRGS, 1998. 173 p.

TAKEBAYASHI, H; MORIYAMA, M. Surface heat budget on green roof and high reflection roof for mitigation of urban heat island. Building and Environment, v.42, n. 8, p. 2971-2979. 2007. Disponível em: http: / /www.sciencedirect.com/science/article /pii/S0360132306001752 Acesso em: 15 set.
2011.

TRIPLEHORN. C.A., JONNSON N.F. Estudo dos insetos. Editora Cengage Learning, São Paulo, 2011. 809 p.

VENDRUSCOLO, S.G. e MENTZ, L. A. Levantamento etnobotânico das plantas utilizadas como medicinais por moradores do bairro Ponta Grossa, Porto Alegre, Rio Grande do Sul, Brasil. Iheringia: série Botânica, Porto Alegre. v. 61, n. 1-2, p. 83-103. 2006. Disponível em: < http://www.fzb.rs.gov.br/publicacoes/iheringi a-botanica/Ih61-p83-103.pdf > Acesso em: 04 dez. 2012.

YANG, J.; YU, Q.; GONG, P. Quantifying air pollution removal by green roofs in Chicago. Atmospheric Environment, v. 42, n. 31, p. 7266-7273. 2008. Disponível em: http://www.sciencedirect.com/science/article /pii/S1352231008006262 Acesso em: 16 set. 2011.

YOUNG, D.A. Taxonomic Study of the Cicadellinae (Homoptera: Cicadellidae): Part. 2 New Word Cicadellini and the Genus Cicadella. The North Carolina Agricultural Experiment Station, 1977. 1135 p.

ZILLER, R.S. Plantas exóticas invasoras a ameaça da contaminação biológica. Revista Ciência Hoje. Rio de Janeiro, v. 30, n 178, p. 77-79, dez. 2001.

The implementation of a quality management system based on the Q tourist quality standard. The case of hotel sector. Service Business, v. 4, n. 3-4, p. 177 - 196, 2010. 